Habituation to contmuous treatment-There was no further reduction in heart rate or blood pressure at rest or during exercise with any drug after eight weeks compared with the results after four weeks' treatment (table II).

Specificity of antihypertensive activity-After transfer to placebo heart rate and blood pressure returned to the pretreatment range in all patients within four weeks. There was no significant change in either variable during the subsequent four weeks with the exception of the systolic pressure at the highest level of exertion $(P<0.05)$ (table II)

\section{Discussion}

These studies clarify some of the debate that has arisen about the comparative antihypertensive effectiveness of different betaadrenoceptor antagonists. During sustained treatment, in doses which produced comparable reductions in exercise tachycardia, all drugs had similar specific blood-pressure lowering activity, greater on systolic than on diastolic pressure, greatest during exercise, and unassociated with habituation.

Therapeutic benefits and disadvantages have been claimed for some of the ancillary pharmacological properties of these drugs. Our studies indicate that such properties are of little importance in relation to their blood-pressure lowering activity in hypertensive patients. Although intrinsic stimulating effects on heart rate were observed with oxprenolol, practolol, and tolamolol, these drugs were equal in antihypertensive effectiveness to those without this property. Cardioselectivity (practolol and metoprolol) likewise did not seem to enhance blood-pressure lowering activity. Tolamolol possesses weak vasodilator activity, ${ }^{7}$ which probably accounted for the postural drop in blood pressure on this drug compared with the lack of such effect with drugs without this property. Nevertheless, this augmented bloodpressure lowering activity on standing did not persist during walking. Hence the antihypertensive activity of all these drugs is predominantly related to their common property of blockade of cardiac sympathetic beta-adrenoceptors, and their ancillary pharmacological properties, with the possible exception of intrinsic vasodilator activity, play little part in this response. These observations confirm other less comprehensive comparisons. ${ }^{*} 9$

Our results also give information on prescribing. The reduction in blood pressure was directly related to the dose of beta-blocking drug - that is, the higher the dose the greater the reduction in blood pressure. A similar dose-response relation- ship was also observed when these drugs were first administered to hypertensive patients. ${ }^{10}$ In our study the final dose of each drug was arbitrarily fixed at the dose which induced a reduction in standing diastolic pressure to $100 \mathrm{~mm} \mathrm{Hg}$ or at a maximum of 16 capsules twice daily. Given these dosage end-points the final comparative doses were relatively narrow, ranging from 5-7 $\mathrm{mg} / \mathrm{kg}$ body weight (with the exception of practolol, $10 \mathrm{mg} / \mathrm{kg}$ ).

A point of possible therapeutic importance was that these drugs had a greater hypotensive effect on systolic than on diastolic pressure and the greatest effect was during exertion. Direct correlation between the height of the systolic pressure and morbidity and mortality from cardiovascular events is now firmly established. ${ }^{11}$ The relation is even closer if exercise and other daily events are taken into account, ${ }^{12}$ and there are feasible biological links to establish a cause-effect relationship between these correlates. ${ }^{13}$ Lowering of resting pressure and the suppression of the pressor response to exercise may therefore be important in preventing vascular complications during longterm treatment of hypertension with these drugs. This, together with the protection of the myocardium from sympathetic stimulation, may be an important therapeutic advantage possessed by these drugs over other antihypertensive agents in preventing the cardiac consequences of hypertensive vascular disease.

UT is a Ciba research fellow and WS a Pfizer research scholar in receipt of a grant from the West Riding Medical Research Trust.

\section{References}

${ }^{1}$ Medical Research Council Annual Report, British Medical Fournal, 1963, $2,178$.

2 Ormrod, R, British Medical fournal, 1968, 2, 7.

3 Thadani, U, et al, British Medical fournal, 1973, 1, 138.

4 Taylor, S H, et al, International fournal of Clinical Pharmacology, 1974, $10,136$.

5 Davidson, C, et al, Circulation, in press.

${ }^{6}$ Duncan, D B, Biometrics, 1955, 11, 1 .

7 Taylor, S H, Singleton, W, and McGibney, D, in preparation.

${ }^{8}$ Leishman, A W D, et al, British Medical fournal, 1970, 4, 342

${ }^{9}$ Morgan, T O, et al, Postgraduate Medical fournal, 1974, 50, 253.

io Singleton, W, et al, Clinical Science and Molecular Medicine, 1975, 48, 18.

${ }^{11}$ Kannel, W B, and Dawber, T B, British fournal of Hospital Practice, 1974 11, 508.

12 Sokolow, M, et al, Circulation, 1966, 34, 279.

${ }_{13}$ Taylor, S H, in Hypertension-Its Nature and Treatment, ed D M Burley, et al. Horsham, Ciba Laboratory Publications, 1975.

\title{
Blood pressure survey in a population of newborn infants
}

\author{
$M$ de SWIET, P FAYERS, E A SHINEBOURNE
}

British Medical fournal, 1976, 2, 9-11

\section{Summary}

Systolic blood pressure in the arm was measured in infants at the ages of 4 to 6 days and 5 to 7 weeks by the

\footnotetext{
Department of Paediatrics, Cardiothoracic Institute and MRC Tuberculosis and Chest Diseases Unit, Brompton Hospital, London SW3 6HP

M DE SWIET, MD, MRCP, senior lecturer

P FAYERS, BSC, statistician

E A SHINEBOURNE, MD, MRCP, consultant paediatric cardiologist
}

\begin{abstract}
Doppler ultrasound technique. At the age of 4 to 6 days the mean blood pressure ( $\pm S E$ of mean) in 469 sleeping infants was $70.7 \pm 0.3 \mathrm{~mm} \mathrm{Hg}$, rising at 5 to 7 weeks to $89.7+0.9 \mathrm{~mm} \mathrm{Hg}$ (in 144 infants). In 252 infants awake at 5 to 7 weeks blood pressure was $96.8 \pm 0.6 \mathrm{~mm} \mathrm{Hg}$. In 391 infants in whom measurements were made on both occasions blood pressure at 4 to 6 days was significantly related to blood pressure at 5 to 7 weeks. Thus those infants with relatively high blood pressures at 4 to 6 days showed a weak tendency to have relatively high blood pressures at 5 to 7 weeks. If this trend continues with age it would suggest that the tendency to develop hypertension may already be demonstrable at the age of 4 to 6 days.
\end{abstract}




\section{Introduction}

Epidemiological studies ${ }^{1}$ have shown that even by the age of 15 years the subsequent course of a person's blood pressure pattern may already be established. Thus people with relatively high blood pressures when first studied at the age of 15 tend to have relatively high blood pressures when restudied 10 years later. Similarly, those with relatively low blood pressures initially have low blood pressures when remeasured. More recently Kass et $a l^{2}$ have provided some evidence to suggest that there is a correlation between blood pressure first measured at the age of 2 years and blood pressure measured four years later.

We have shown that blood pressure can be measured accurately and non-invasively in neonates using a Doppler ultrasound technique, ${ }^{3}$ and we have characterised some of the factors that influence blood pressure variability in the neonatal period. ${ }^{* 5}$ We have now embarked on an epidemiological study of the normal range of blood pressure in infancy. We will also investigate whether measurements of systolic blood pressure in infancy or childhood make it possible to predict the course of the person's blood pressure in later years.

We present here the results of measurements in 391 infants whose blood pressures were measured at both 4 to 6 days and 5 to 7 weeks. Measurements were made at 4 to 6 days only in an additional 109 infants.

\section{Methods}

Blood pressure measurement-Systolic blood pressure in the arm was measured at home $\left(30^{\circ}\right)$ or in hospital $\left(70^{\circ}{ }_{0}\right)$ when the subjects were 4 to 6 days old, and again at home when they were 5 to 7 weeks old. Measurements were made by nurses specially trained in using the Parkes Doppler ultrasound system $^{3}$ and the random zero sphygmomanometer. ${ }^{6}$ A $4-\mathrm{cm}$ cuff was used, the largest cuff that would encircle the arm. All blood pressure measurements were repeated three times and the mean was calculated for subsequent analysis. The mothers' systolic and diastolic blood pressures were also measured on the same occasions using the random zero sphygmomanometer in the conventional manner. Diastolic blood pressure was taken when the sounds were muffled. The infant's level of consciousness was noted and defined as awake if the eyes were open at the onset of blood pressure measurement or asleep if the eyes were closed. ${ }^{4}$ No measurements were made during crying or feeding.

The sample-All babies born after 38 weeks'.gestation at Farnborough Hospital, Kent, between 1 May and 1 December 1975 whose mothers lived in the London Borough of Bromley were included in this study. Blood pressure was measured in 500 infants when they were 4 to 6 days old. We were unable to make measurements in a further 35 infants: the parents of 22 refused to co-operate, three had died by four days, and 10 were too ill for meaningful blood pressure measurement. Blood pressure measurements were repeated at the age of 5 to 7 weeks. By 1 December 1975412 infants were at least 5 weeks old and blood pressure measurements had been made on 396. A further six infants were withdrawn because of failure of parental co-operation; two families were on extended holiday; and eight infants had acute illnesses, mainly upper respiratory tract infections.

\section{Results}

The distribution of upper limb systolic blood pressure at the age of 4 to 6 days is shown in fig 1 . Mean systolic blood pressure $( \pm \mathrm{SE}$ of mean) in 469 sleeping infants was $70.7 \pm 0.3 \mathrm{~mm} \mathrm{Hg}$. In 31 babies who were awake the mean systolic blood pressure was $76.9 \pm 1.8$ $\mathrm{mm} \mathrm{Hg}$.

The distribution of upper limb systolic blood pressure at 5 to 7 weeks is shown in fig 2. Mean systolic blood pressure in 252 infants who were awake was $96.8 \pm 0.6 \mathrm{~mm} \mathrm{Hg}$, which was significantly greater $(P<0.001)$ than the mean systolic blood pressure in 144 sleeping infants $(89 \cdot 7 \pm 0.9 \mathrm{~mm} \mathrm{Hg})$. Systolic blood pressures asleep and awake at 5 to 7 weeks were both significantly greater $(\mathbf{P}<0.001)$ than the corresponding systolic blood pressure asleep and awake at 4 to 6 days.

The blood pressure of babies awake at 5 to 7 weeks was adjusted for wakefulness by subtracting $7 \cdot 1 \mathrm{~mm} \mathrm{Hg}$, the mean difference in blood

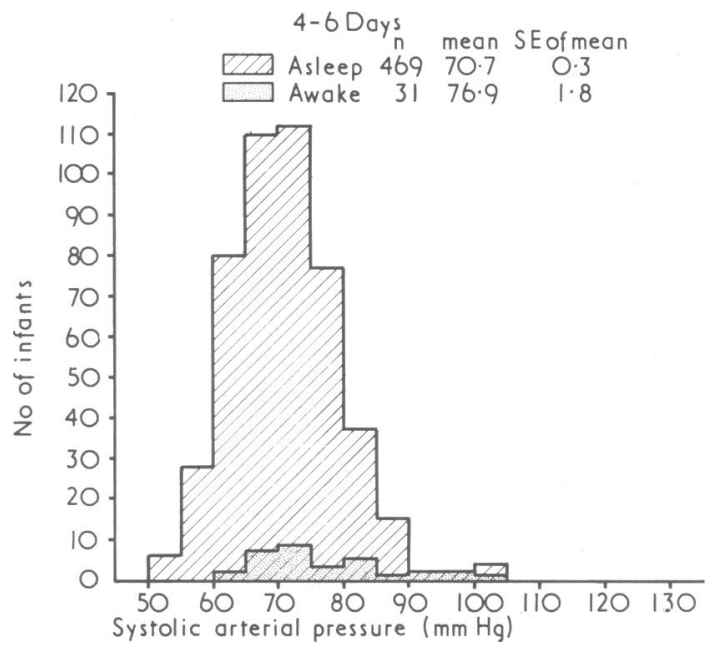

FIG 1-Distribution of systolic arterial pressure in 500 infants aged 4 to 6 days asleep and awake.

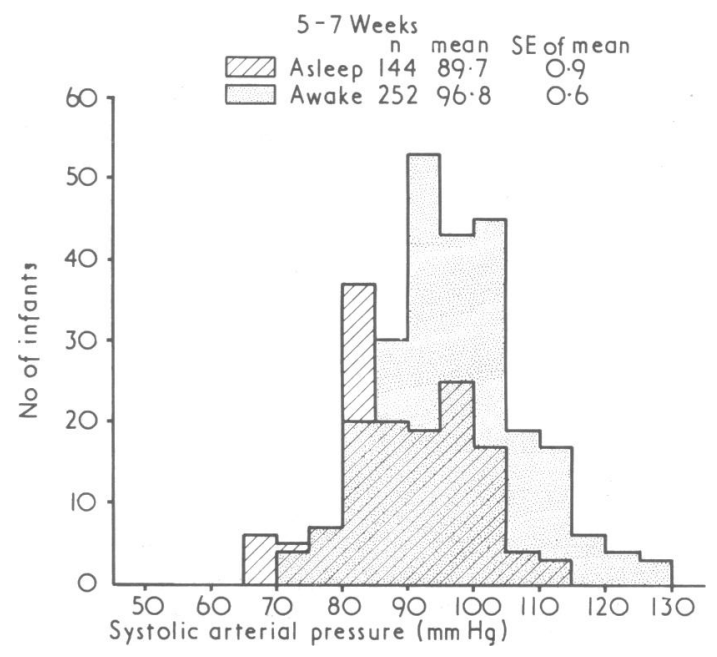

FIG 2-Distribution of systolic arterial pressure in 396 infants aged 5 to 7 weeks asleep and awake.

pressure between sleeping and waking babies at 5 to 7 weeks. A $N$ similar adjustment was made for the 31 babies awake at four days. The $D$ adjusted blood pressures at 5 to 7 weeks were then compared with adjusted blood pressures at 4 to 6 days. They were significantly correlated $(r=0.20 ; P<0.001)$.

\section{Discussion}

There have been many reports of casual measurements of $\stackrel{\mathbb{D}}{\oplus}$ blood pressure in the neonatal period, ${ }^{7-9}$ but we are unaware of 0 any studies in which blood pressure has been measured in $\vec{O}$ an unselected population of neonates with subsequent measure- $\stackrel{\mathbb{\Phi}}{\Omega}$ ments as infants become older. We have shown an increase in $\mathbb{\mathbb { Q }}$ blood pressure between 4 days and 6 weeks. This is in keeping with the well-established increase in blood pressure with age in older age groups. ${ }^{1}$

Children awake have higher blood pressures than children asleep. ${ }^{4}$ We measured blood pressure at the ages of 4 to 6 days $\frac{\bar{a}}{\partial}$ and 5 to 7 weeks because of the practical difficulty of measuring? blood pressure at a precise age in a large population. We had found that blood pressure rises most rapidly in the first four days after birth. ${ }^{4}$ Therefore we did not make measurements earlier than 4 days, since any variability in the age at which the 
infants were studied before 4 days would cause extra variability in blood pressure measurement.

In this study the mean systolic blood pressure increased by $1.5 \mathrm{~mm} \mathrm{Hg}$ /day within the short period of 4 to 6 days and $0.5 \mathrm{~mm} \mathrm{Hg} /$ week within the period of 5 to 7 weeks. Nevertheless, these increases were small compared with the overall rise in blood pressure $(20 \mathrm{~mm} \mathrm{Hg}$ ) between 4 to 6 days and 5 to 7 weeks.

We used the Parkes Doppler ultrasound system, which, we have found, measures only systolic blood pressure. ${ }^{3}$ Systolic and diastolic blood pressures are highly correlated, however, and, furthermore, systolic blood pressure is a better predictor of subsequent cardiovascular morbidity than diastolic blood pressure. ${ }^{10}$

We have found a highly significant, though weak, relation between infants' blood pressures at the age of 4 to 6 days and those at the age of 5 to 7 weeks. The relation is not strong, probably because of the high degree of variability of blood pressure that we have previously observed in the neonatal period.

Kass et al have shown that the blood pressure at the ages of 2 to 14 years is related to blood pressure when measured again four years later, at the ages of 6 to 18 years." Also, a familial aggregation of blood pressure may be shown by the age of 2 years. ${ }^{11}$ If our follow-up studies confirm that the relation that we have already found is maintained until the age of 2 years, it would suggest that the blood pressures of some people may be predetermined by the time they are 4 to 6 days old and that the tendency to hypertension may already be manifest by that age.

It is a pleasure to acknowledge the generous financial support of the Medical Research Council to Michael de Swiet and to the nurses, D Johnson, J Mitchell, F Smith, and E Szewvzyk; without their enthusiasm none of the measurements would have been made. We are grateful to Dr P Wallis and Dr P Swift for permission to study patients under their care, to $\mathrm{Dr} W$ Fox for invaluable criticism, and to Mrs $S$ Cowley for secretarial help.

\section{References}

Mial, W E, and Lovell, A G, British Medical fournal, 1967, 2, 660.

Zinner, S H, et al, American fournal of Epidemiology, 1974, 100, 437.

${ }^{3}$ Elseed, A M, Shinebourne, E A, and Joseph, M C, Archives of Disease in Childhood, 1973, 48, 932.

+ Swiet, $M$ de, Fancourt, R, and Peto, J, Clinical Science and Molecular Medicine, 1975, 49, 557 .

'Swiet, M de, Peto, J, and Shinebourne, E A, Archives of Disease in Childhood, 1974, 49, 734

Wright, B M, and Dore, C F, Lancet, 1970, 1, 337.

Modanlou, H, et al, fournal of Paediatrics, 1974, 85, 553.

${ }^{8}$ Moss, A J, and Adams, F H, American fournal of Diseases of Children, 1964, 107, 489

${ }^{9}$ Kirkland, R T, and Kirkland, J L, fournal of Pediatrics, 1972, 80, 52.

${ }^{10}$ Kannel, W B, Gordon, T, and Schwartz, M J, American fournal of Cardiology, 1971, 27, 335.

11 Kass, E H, and Zinner, S H, Millbank Memorial Fund Quarterly Bulletin, 1969, 47, 143.

\title{
Adrenal aldosterone-producing adenoma: use of colonic potential in diagnosis and subtraction scanning technique for localisation
}

\author{
K E BRITTON, T J GOODWIN, W S PEART, M E SNELL
}

British Medical fournal, 1976, 2, 11-14

\section{Summary}

Primary hypoaldosteronism is a potentially curable cause of hypertension, and much interest has been shown in methods of diagnosing the associated hypokalaemic hypertension and localising the adrenal adenoma. In two patients the diagnosis of primary aldosteronism was confirmed by colonic potential measurement and the adenoma localised by a new subtraction technique for early adrenal imaging applied to the use of ${ }^{131} \mathrm{I}-19$ iodocholesterol. Both patients underwent adrenalectomy and in each case an adenoma was removed. Blood pressure and electrolyte levels returned to normal after operation. In one patient bilateral adrenal phlebography

Department of Nuclear Medicine, Middlesex Hospital Medical School, London W1N 8AA

K E BRITTON, MD, MRCP, consultant in nuclear medicine

Mount Vernon Hospital, Northwood, Middlesex HA6 2RN

T J GOODWIN, MRCP, consultant physician

St Mary's Hospital, London W2

W S PEART, MD, FRCP, FRS, professor of medicine

M E SNELL, FRCS, consultant surgeon had failed to show the tumour, and sampling of aldosterone concentrations in the adrenal veins had been unsatisfactory.

\section{Introduction}

Primary hyperaldosteronism was first recognised as a clinical syndrome 22 years ago. ${ }^{1}$ High blood pressure, persistently low concentrations of plasma potassium, and high plasma sodium concentrations were the main clinical and biochemical features in the first patient. A large adrenal adenoma was subsequently removed and the blood pressure and plasma electrolytes reverted to normal levels. Since this report there has been considerable interest in the diagnosis of hypokalaemic hypertension associated with primary hyperaldosteronism and in methods for localising the adenoma. We describe the clinical and biochemical features of two patients in whom the diagnosis was confirmed by colonic measurement and the adenoma was localised by a new subtraction technique for early adrenal imaging applied to the use of ${ }^{131}$ I-19-iodocholesterol.

\section{Methods}

PROTOCOL FOR MEASUREMENT OF COLONIC POTENTIAL

Colonic potential difference was determined by the methods of Edmonds and Godfrey ${ }^{2}$ and Efstratopoulos $e t$ al ${ }^{3}$ using a probe electrode of perspex tubing containing $4 \%$ agar jelly with $150 \mathrm{mmol} / \mathrm{l}$ 